

\title{
Thermal conductivity of nano-layered systems due to surface phonon-polaritons
}

Jose Ordonez-Miranda, Laurent Tranchant, Yann Chalopin, Thomas Antoni, Sebastian Volz

\section{- To cite this version:}

Jose Ordonez-Miranda, Laurent Tranchant, Yann Chalopin, Thomas Antoni, Sebastian Volz. Thermal conductivity of nano-layered systems due to surface phonon-polaritons. Journal of Applied Physics, 2014, pp.6. 10.1063/1.4864430] . hal-01134042

\section{HAL Id: hal-01134042}

https://hal-centralesupelec.archives-ouvertes.fr/hal-01134042

Submitted on 24 Mar 2015

HAL is a multi-disciplinary open access archive for the deposit and dissemination of scientific research documents, whether they are published or not. The documents may come from teaching and research institutions in France or abroad, or from public or private research centers.
L'archive ouverte pluridisciplinaire HAL, est destinée au dépôt et à la diffusion de documents scientifiques de niveau recherche, publiés ou non, émanant des établissements d'enseignement et de recherche français ou étrangers, des laboratoires publics ou privés. 


\title{
Thermal conductivity of nano-layered systems due to surface phonon-polaritons
}

\author{
Jose Ordonez-Miranda, ${ }^{1}$ Laurent Tranchant, ${ }^{1}$ Yann Chalopin, ${ }^{1}$ Thomas Antoni, ${ }^{1,2}$ \\ and Sebastian Volz ${ }^{1, a)}$ \\ ${ }^{1}$ Laboratoire d'Énergétique Moléculaire et Macroscopique, Combustion, UPR CNRS 288, \\ École Centrale Paris, Grande Voie des Vignes, 92295 Châtenay-Malabry, France \\ ${ }^{2}$ École Centrale Paris, Laboratoire de Photonique Quantique et Moléculaire, CNRS (UMR 8537), \\ École Normale Supérieure de Cachan, Grande Voie des Vignes, F-92295 Châtenay-Malabry cedex, France
}

(Received 18 December 2013; accepted 23 January 2014; published online 6 February 2014)

\begin{abstract}
The effective thermal conductivity of a layered system due to the propagation of surface phonon-polaritons is studied. We analytically demonstrate that the thermal conductivity of a set of nanolayers can be described as one of a single layer with an effective permittivity, which does not ordinarily appear in nature and depends on the permittivities and thicknesses of the individual components. For a two-layer system of $\mathrm{SiO}_{2}$ and $\mathrm{BaF}_{2}$ surrounded by air, it is shown that: (i) the propagation length of surfaces phonon-polaritons can be as high as $3.3 \mathrm{~cm}$ for a $200 \mathrm{~nm}$-thick system. (ii) The thermal conductivity of the system with total thickness of $50 \mathrm{~nm}$ is $3.4 \mathrm{~W} / \mathrm{m} \cdot \mathrm{K}$, which is twice that of a single layer of $\mathrm{SiO}_{2}$, at $500 \mathrm{~K}$. Higher values are found for higher temperatures and thinner layers. The results show that an ensemble of layers provides more channels than a single layer for the propagation of surface phonon-polaritons and therefore for the enhancement of the thermal conductivity of common polar materials. (C) 2014 AIP Publishing LLC. [http://dx.doi.org/10.1063/1.4864430]
\end{abstract}

\section{INTRODUCTION}

The blossoming of nanotechnology through the miniaturization of devices with enhanced rates of operation requires a profound understanding of their thermal performance. This is particularly critical in nanomaterials, which are susceptible to overheating, due to the reduction of their effective thermal conductivity as their size is scaled-down. Over the past few years, the surface phonon-polaritons (SPPs) ${ }^{1-3}$ have been proposed as potential energy carriers to offset this reduction, especially in materials with low phonon thermal conductivity, as is the case of the amorphous $\mathrm{SiO}_{2}$ and crystalline $\mathrm{SiC}$. Various research groups have shown that the SPPs have promising applications on the thermal performance of nanoscale devices, ${ }^{4-6}$ radiative heat transfer, ${ }^{7-10}$ high-density infrared data storage, ${ }^{11}$ surface infrared absorption, ${ }^{12}$ coherent thermal emission, ${ }^{13}$ and photonics. ${ }^{14,15}$ SPPs are electromagnetic waves that propagate along the interface between two materials, and therefore their energy transport is expected to significantly increase as the system size reduces to nanoscales, due to the predominance of the surface effects at these size scales. ${ }^{4,6}$

One key difference of SPPs with respect to phonons is their comparatively long propagation length, which can be of the order of a few centimeters on thin films. ${ }^{4-6}$ Based on the Boltzmann transport equation, Chen et al. ${ }^{4}$ showed that the SPP thermal conductivity of a $40 \mathrm{~nm}$-thick thin film of amorphous $\mathrm{SiO}_{2}$ suspended in air is $4 \mathrm{~W} / \mathrm{m} \cdot \mathrm{K}$ at $500 \mathrm{~K}$. This numerical result represents an increase of about $100 \%$ over the intrinsic phonon thermal conductivity, has been confirmed by the fluctuation-dissipation theorem, ${ }^{16}$ and it is

\footnotetext{
a) Author to whom correspondence should be addressed. Electronic mail: sebastian.volz@ecp.fr
}

consistent with experimental data for the propagation length. ${ }^{5}$ More recently and under a fully analytical approach, we showed that a substrate with a permittivity slightly different than that of the superstrate of a nano thin film can generate large propagation lengths and therefore significantly enhance its SPP thermal conductivity to values even higher than those previously reported. ${ }^{4}$ The propagation of SPPs along multilayered materials has been also theoretically studied $^{17-20}$ and their long propagation length has been experimentally observed. ${ }^{21}$ However, to date and to our knowledge, the quantification of the SPP thermal conductivity of these layered systems has not been explored yet. Taking into account that the absorption of energy by a layer is driven by its permittivity and that the SPP thermal conductivity depends strongly on the film thickness, it is expected that an ensemble of thin films with different permittivities and thicknesses can provide more variables and channels than a single film to control and optimize the SPP energy transport through an increase of the number of interfaces.

The purpose of this work is to analyze, quantify, and optimize the thermal conductivity of a layered system due to the propagation of SPPs. For layers with a thickness comparable or smaller than a hundred of nanometers, explicit expressions for the effective permittivity of the layers and their SPP thermal conductivity as a function of total thickness are obtained. Both of these relations provide clear physical insights on how the heat transport depends on the permittivity of the layers and their thickness.

\section{THERMAL CONDUCTIVITY MODELING}

Let us consider a layered system supporting the propagation of SPPs, as shown in Fig. 1(a). Each layer has a 

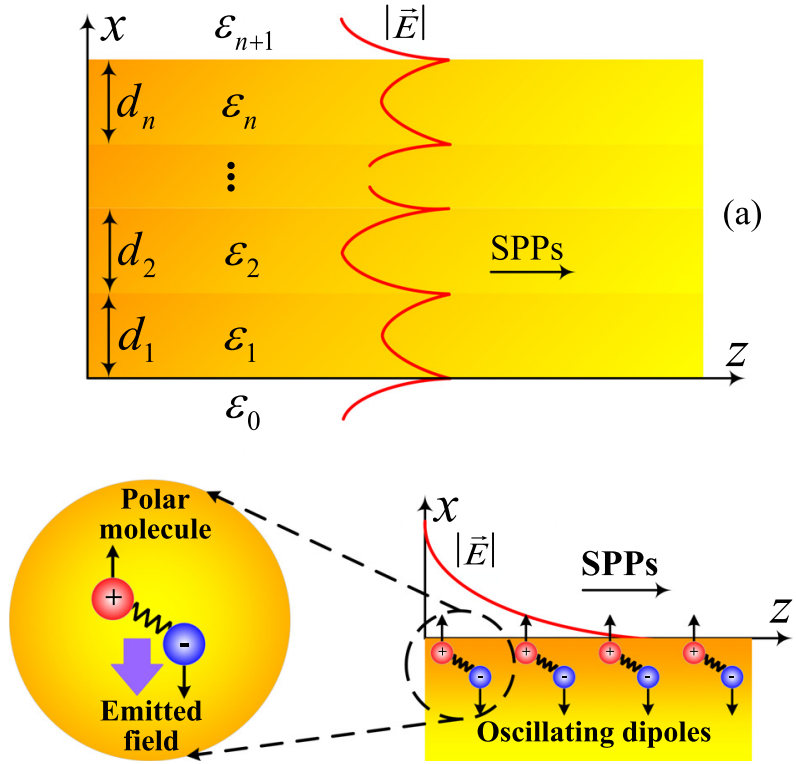

(b)

FIG. 1. (a) Cross-section of the layered system under consideration and (b) generation of SPPs by the fluctuation of electrical dipoles.

frequency-dependent permittivity $\varepsilon_{n}$, for $n=1,2, \ldots, N$. The surrounding media on either sides have constant permittivities $\varepsilon_{0}$ and $\varepsilon_{n+1}$, as is the case of air or $\mathrm{KBr}$, within a wide range of frequency. ${ }^{22}$ The SPPs are generated within the layers by the fluctuation of microscopic electrical dipoles, which under a thermal excitation, oscillate and emit an electric field $\vec{E}$. This field induces the excitation of neighboring surface electrical dipoles, which keep the propagation of the field (SPP) along the layers, as shown in Fig. 1(b). These electrical dipoles are spontaneously present in polar materials, such as $\mathrm{SiO}_{2}$ and $\mathrm{SiC}$. The SPP dispersion relation is necessary to determine the thermal conductivity of this system. ${ }^{6}$ This relation can be found by solving the Maxwell equations under proper boundary conditions for the transverse magnetic polarization, which is required for the existence of SPPs. ${ }^{4}$ For two layers $(n=2)$, the following dispersion relation for the wave vector $\beta$ along the $z$ axis is obtained:

$$
\frac{\tanh \left(p_{1} d_{1}\right)+\alpha_{01}}{1+\alpha_{01} \tanh \left(p_{1} d_{1}\right)}=-\alpha_{21} \frac{\tanh \left(p_{2} d_{2}\right)+\alpha_{32}}{1+\alpha_{32} \tanh \left(p_{2} d_{2}\right)},
$$

where $\alpha_{n m}=\varepsilon_{n} p_{m} / \varepsilon_{m} p_{n}$, the transverse wave vectors $p_{n}$ along the $x$ axis are given by $p_{n}^{2}=\beta^{2}-\varepsilon_{n} k_{0}^{2}, k_{0}=\omega / c, \omega$ is the excitation frequency, and $c$ is the speed of light in vacuum. Note that for $\varepsilon_{1}=\varepsilon_{2}$, Eq. (1) reduces to our previous result for one layer. ${ }^{6}$

Equation (1) can significantly be simplified for thin layers $\left(\left|p_{n}\right| d_{n} \ll 1\right)$, which is of interest in this work to enhance the SPP propagation along the layers. For nano layers of $\mathrm{SiO}_{2}$ and $\mathrm{SiC}$, for instance, this condition is well satisfied for $d_{n} \leq 300 \mathrm{~nm}$. In this case and under a first-order approximation on $d_{n}$, Eq. (1) reduces to the symmetric equation

$$
\frac{p_{1}^{2}}{\varepsilon_{1}} d_{1}+\frac{p_{2}^{2}}{\varepsilon_{2}} d_{2}+\frac{p_{0}}{\varepsilon_{0}}+\frac{p_{3}}{\varepsilon_{3}}=0 .
$$

Note that for thin layers $\left(\left|p_{n}\right| d_{n} \ll 1\right) \beta \rightarrow \sqrt{\varepsilon_{0}} k_{0}$, which implies that $p_{0} \rightarrow 0$. Therefore, $p_{n}^{2}=p_{0}^{2}+\left(\varepsilon_{0}-\varepsilon_{n}\right) k_{0}^{2}$ $\approx\left(\varepsilon_{0}-\varepsilon_{n}\right) k_{0}^{2}$ and Eq. (2) yields

$$
\begin{gathered}
\frac{p_{0}+\frac{p_{3}}{\varepsilon_{0}}}{\varepsilon_{3}}=d\left(1-\frac{\varepsilon_{0}}{\varepsilon}\right) k_{0}^{2}, \\
\frac{d}{\varepsilon}=\frac{d_{1}}{\varepsilon_{1}}+\frac{d_{2}}{\varepsilon_{2}},
\end{gathered}
$$

where $d=d_{1}+d_{2}$. Equation (3a) shows that the two-layer system acts like a single layer with an effective permittivity $\varepsilon$. This result was previously derived for the propagation of interface waves along periodic superlattices made up of uniaxial crystals, ${ }^{23,24}$ and it holds for the permittivity components along the optical axes only. Here, we are showing that Eq. (3b) also applies for isotropic polar layers. When the two surrounding media are equal $\varepsilon_{0}=\varepsilon_{3}\left(p_{0}=p_{3}\right)$, Eq. (3a) has the same shape than our corresponding previous result for one layer. ${ }^{6}$ Hence, based on the analytical expressions for the real part $\beta_{R}$ of $\beta=\beta_{R}+i \beta_{I}$ and propagation length $\Lambda=1 /\left(2 \beta_{I}\right)$ derived for a single layer, ${ }^{6}$ the SPP thermal conductivity of the two-layer system can be written as

$$
\kappa=\frac{A}{d^{3}}+\frac{B}{d},
$$

where

$$
\begin{gathered}
A=\frac{1}{2 \pi} \int_{0}^{\infty} \frac{\hbar \omega}{\xi k_{0}^{2}} \frac{|\varepsilon|^{2}}{\varepsilon_{0}^{2} \varepsilon_{I}} \frac{\partial f}{\partial T} d \omega \\
B=\frac{1}{8 \pi} \int_{0}^{\infty} \hbar \omega\left(\frac{\xi}{\varepsilon_{0} \varepsilon_{I}}-\frac{\varepsilon_{0} \varepsilon_{I}}{\xi}\right) \frac{\partial f}{\partial T} d \omega,
\end{gathered}
$$

$f$ being the Bose-Einstein distribution function, $T$ is the temperature, $\varepsilon=\varepsilon_{R}-i \varepsilon_{I}$ and $\xi=|\varepsilon|^{2}-\varepsilon_{0} \varepsilon_{R}$, which must be positive for any $\omega>0$, to guarantee the propagation of SPPs $(\Lambda>0) .{ }^{6}$ Within the thin film limit $\left(\left|p_{n}\right| d_{n} \ll 1\right)$, the simple Eq. (4) establishes that the increase of the SPP thermal conductivity as the film thickness decreases is fully driven by the material-dependent parameters $A$ and $B$. The smaller values of the layer thicknesses $d_{n}$ are set by the surface roughness height and peak-to-peak distance, which are typically of the order of $1 \mathrm{~nm}^{4}$

For $\varepsilon_{0} \neq \varepsilon_{3}$, as is the case of thin films deposited over a substrate, the solution of Eq. (3a) for $p_{0}$ is

$$
p_{0}=\frac{d}{2}\left(1-\frac{\varepsilon_{0}}{\varepsilon}\right) \varepsilon_{0} k_{0}^{2}-\frac{\Delta}{2 d} \frac{\varepsilon_{0}}{\varepsilon_{3}^{2}} \frac{\varepsilon}{\varepsilon-\varepsilon_{0}},
$$

where $\Delta=\varepsilon_{0}-\varepsilon_{3}$. Note that the first term of Eq. (6) is the same than Eq. (3a) for the symmetric case $\left(\varepsilon_{0}=\varepsilon_{3}\right)$, while the second term contains the contribution of the asymmetry $\left(\varepsilon_{0} \neq \varepsilon_{3}\right)$ of the surrounding media. Equation (6) coincides with our previous result for a single layer between two media of different permittivity, provided that its permittivity $\varepsilon$ is given by Eq. (3b). ${ }^{6}$ Therefore, the thermal conductivity of this asymmetric system can also be determined with the analytical expression previously derived. ${ }^{6}$ 
The generalization of Eq. (3b) for a system of $n>2$ layers can easily be done by following a similar procedure as the one presented for the two-layer system. The final and expected result is

$$
\frac{d}{\varepsilon}=\frac{d_{1}}{\varepsilon_{1}}+\frac{d_{2}}{\varepsilon_{2}}+\ldots+\frac{d_{n}}{\varepsilon_{n}},
$$

where $d=d_{1}+d_{2}+\ldots+d_{n}$. In this case, the validity of Eqs. (3a) and (6) holds, provided that the effective permittivity of the layers is given by Eq. (7) and $\varepsilon_{3} \rightarrow \varepsilon_{n+1}$. Note that the effective permittivity $\varepsilon$ does not depend on the absolute thicknesses of the layers, but rather on their relative values.

\section{RESULTS AND DISCUSSIONS}

The SPP contribution to the effective thermal conductivity of the layered system shown in Fig. 1(a) is quantified and analyzed in this section. Special emphasis is put on the effects of the layers' size and permittivities of the surrounding media.

Figure 2 shows the frequency dependence of the real $\left(\varepsilon_{R}\right)$ and imaginary $\left(\varepsilon_{I}\right)$ parts of the permittivity of $\mathrm{SiO}_{2},{ }^{6,22}$ in comparison with the effective permittivity of two layers of $\mathrm{SiO}_{2}$ and $\mathrm{BaF}_{2}$ with equal thickness. The first of these materials is amorphous and opaque, while the second one is a transparent crystal with permittivity $\varepsilon_{1}=2 .{ }^{22}$ Note that the peaks of $\varepsilon_{I}$ for the two-layer system are smaller and sharper than the ones of a single layer of $\mathrm{SiO}_{2}$. This indicates that the energy absorption by $\mathrm{SiO}_{2}$ reduces when it is in contact with $\mathrm{BaF}_{2}$ and therefore the SPP energy transport is expected to be more efficient along the $\mathrm{SiO}_{2} / \mathrm{BaF}_{2}$ system than the one through $\mathrm{SiO}_{2}$. On the other hand, the reduction of the peaks of $\varepsilon_{R}$ is associated with a modification of the dispersion relation of SPPs. Given that we are considering nano layers $\left(\beta \rightarrow \sqrt{\varepsilon_{0}} k_{0}\right)$, these modifications are expected to be small.

The normalized real $\beta_{R}$ and imaginary $\beta_{I}$ parts of the wave vector $\beta$ along the SPP propagation direction are shown in Fig. 3(a), at each frequency point shown in Fig. 2. For the $\mathrm{SiO}_{2} / \mathrm{BaF}_{2}$ system, both $\beta_{R}$ and $\beta_{I}$ reduce significantly in comparison with the corresponding ones of a single layer of $\mathrm{SiO}_{2}$. The reduction of $\beta_{I}$ implies the increase of the

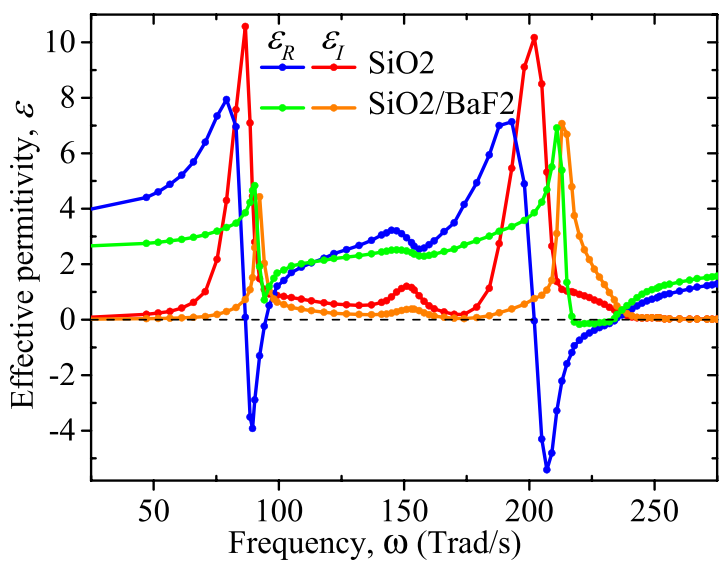

FIG. 2. Real $\left(\varepsilon_{R}\right)$ and imaginary $\left(\varepsilon_{I}\right)$ parts of the effective permittivity of two layers as a function of frequency. Calculations were performed for layers of $\mathrm{SiO}_{2}$ and $\mathrm{BaF}_{2}$ with equal thickness.
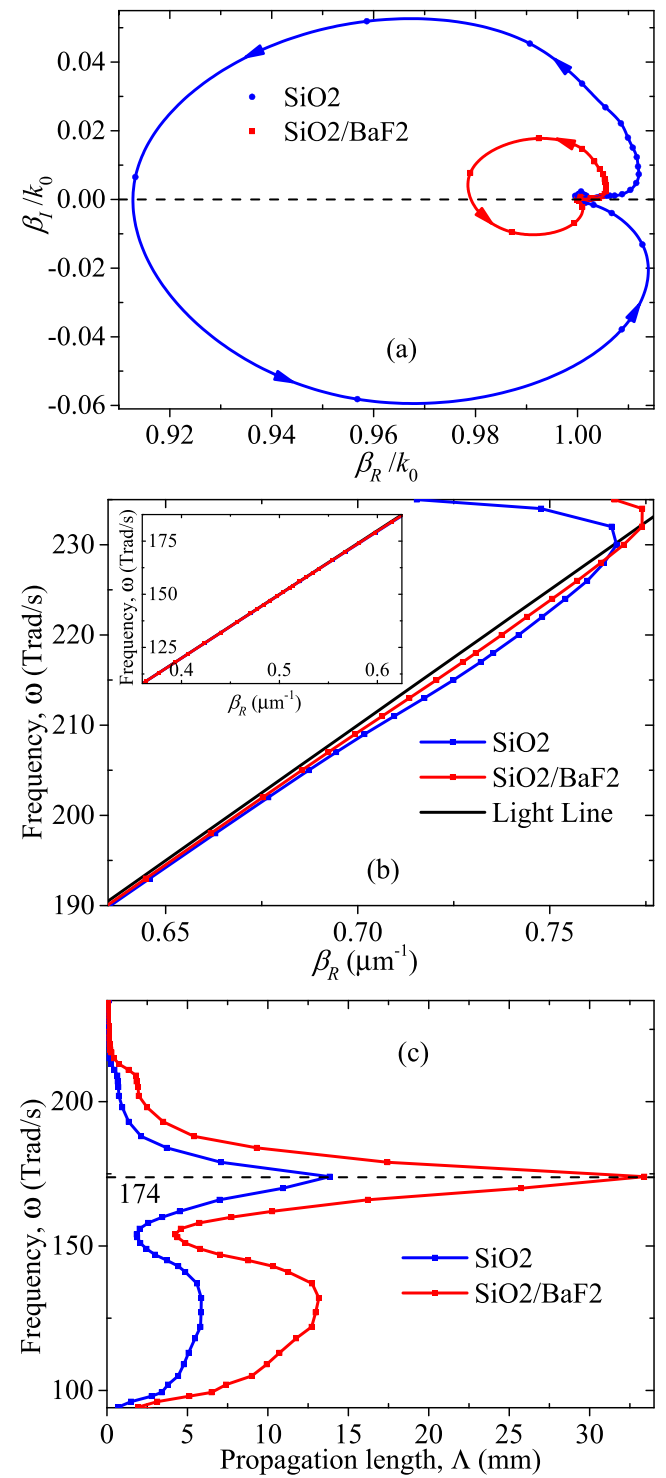

FIG. 3. (a) Contour plot of the complex wave vector $\beta=\beta_{R}-i \beta_{I}$, (b) dispersion relation, and (c) propagation length as a function of frequency, for the $\mathrm{SiO}_{2} / \mathrm{BaF}_{2}$ system surrounded by air $\left(\varepsilon_{0}=\varepsilon_{3}=1\right)$. Calculations were done for $d=200 \mathrm{~nm}$.

SPP propagation length $\Lambda=1 / 2 \beta_{I},{ }^{4,6}$ and hence an enhancement of the energy transport by SPPs. The range of frequencies for which $\beta_{I}>0$ determines the condition for the propagation of SPPs. The dispersion relation and propagation length of SPPs traveling along the $\mathrm{SiO}_{2}$ and $\mathrm{SiO}_{2} / \mathrm{BaF}_{2}$ layers are shown in Figs. 3(a) and 3(b), respectively. The displayed range of frequencies is determined by the conditions of existence $\left(\operatorname{Re}\left(p_{n}\right)>0\right.$, for $\left.n=0,1,2\right)$ and propagation $(\Lambda>0)$ of SPPs. Note that for both the single and two layer system, $\beta_{R}$ is rather close to the light line, especially at low frequencies. This indicates a photon-like nature of the SPPs. As the frequency increases, the dispersion relation separates from the light line and tends to a phonon-like behavior. This behavior strengthens when the film thickness increases. The corresponding propagation lengths in Fig. 3(c) take larger values at the frequencies where the absorption of energy is minimal (Fig. 2). For the single- and two-layer systems, the largest propagation length 
occurs at $174 \mathrm{Trad} / \mathrm{s}$ and their values are $13.8 \mathrm{~mm}$ and $33.4 \mathrm{~mm}$, respectively. This sizeable increase of the propagation length is due to the significant reduction of the energy absorption, as shown in Fig. 2. Therefore, the $\mathrm{SiO}_{2} / \mathrm{BaF}_{2}$ system of two layers is more efficient for the SPP propagation than the single layer of $\mathrm{SiO}_{2}$.

Fig. 4(a) shows that the real parts $\operatorname{Re}\left(p_{n}\right)$ of the transverse wave vectors inside and outside the $\mathrm{SiO}_{2} / \mathrm{BaF}_{2}$ system are positive, and take larger values inside the $\mathrm{SiO}_{2}$ layer, due to its relatively high absorption. The larger the values of $\operatorname{Re}\left(p_{n}\right)$, the more concentrated is the electromagnetic field along the interface of the layers. The fact that $\operatorname{Re}\left(p_{n}\right)>0$ establishes the existence of SPPs, which propagate over the distance shown in Fig. 3(c), in a broad band of frequencies. This is further confirmed by the energy distribution through the Poynting vector shown in Fig. 4(b). The energy flux propagating inside the $\mathrm{SiO}_{2}$ and $\mathrm{BaF}_{2}$ layers is close to zero and negligible in comparison with that outside of them, due to the very small absorption by the surrounding air. The high concentration of energy at the interface enables the propagation of SPPs along the $\mathrm{SiO}_{2} / \mathrm{BaF}_{2}$ system. As a result of the coupling of the electromagnetic fields traveling along the interfaces, the energy flux increases as the film thickness decreases. This indicates that the SPP thermal conductivity should increase as the film thickness reduces, which is consistent with Eq. (4).
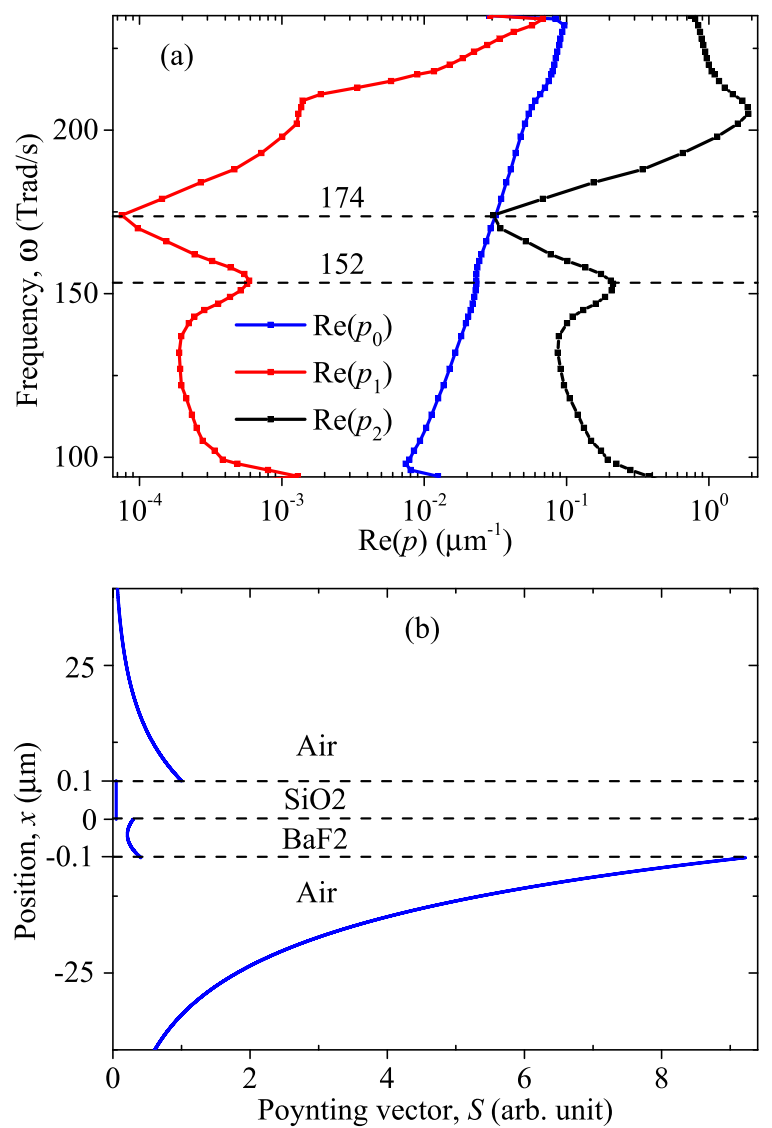

FIG. 4. Frequency dependence of $\operatorname{Re}\left(p_{n}\right)$ inside and outside a $200 \mathrm{~nm}$-thick system of $\mathrm{SiO}_{2} / \mathrm{BaF}_{2}$ surrounded by air. (b) Poynting vector distribution at $x=0$ and $\omega=174 \mathrm{Trad} / \mathrm{s}$, for SPPs propagating along the $z$ axis. The vertical axis scale was lengthened 50 times between -0.1 and $0.1 \mu \mathrm{m}$.
The integrand of the SPP thermal conductivity $\kappa$ defined in Eq. (4) as a function of the frequency is shown in Fig. 5(a), for SPPs propagation along the single- and twolayer systems of $\mathrm{SiO}_{2}$ and $\mathrm{SiO}_{2} / \mathrm{BaF}_{2}$, respectively. Note that the oscillatory behavior of these spectra is determined by the permittivity of the absorbing $\mathrm{SiO}_{2}$, and their maxima coincide with the corresponding ones of the propagation lengths shown in Fig. 3(c). The major contributions to $\kappa$ (area under the curve) arise from the vicinity of these peaks, as shown by the cumulative thermal conductivity in Fig. 5(b). The increase of $\kappa$ exhibits an inflection point at the frequency where the maxima of $\Lambda$ occur. $\kappa$ increases as the total thickness of the layers decreases and it can be much larger than the phonon counterpart of $\mathrm{SiO}_{2}$ for a thickness smaller than $175 \mathrm{~nm}$, as shown in Fig. 5(c). It is clear that $\kappa$ becomes significant for nano thin films and negligible for micro thin films, as found previously for single layers. ${ }^{4,6}$ For a given


FIG. 5. (a) Thermal conductivity spectrum, (b) its cumulative values for $d=100 \mathrm{~nm}$, and (c) thermal conductivity as a function of the total thickness for $T=500 \mathrm{~K}$. Calculations were performed for the layers of $\mathrm{SiO}_{2}$ and $\mathrm{SiO}_{2} / \mathrm{BaF}_{2}$. 
total thickness, $\kappa$ is significantly higher for the $\mathrm{SiO}_{2} / \mathrm{BaF}_{2}$ system than that of the $\mathrm{SiO}_{2}$ layer, due to the comparatively smaller absorption and larger propagation length shown in Figs. 2 and 3(c), for the two-layer system. This enhancement increases for smaller thicknesses, such that for $d=50 \mathrm{~nm}$, $\kappa=3.4 \mathrm{~W} / \mathrm{m} \cdot \mathrm{K}$, which is $100 \%$ higher than the SPP thermal conductivity of the $\mathrm{SiO}_{2}$ layer and its phonon counterpart, at $T=500 \mathrm{~K}$. This shows that the layered systems have the potential not only to enhance their phonon thermal conductivity but also the SPP one of single layers. It is important to keep in mind that the source of the high SPP thermal conductivity is the propagation length at wavevectors close to the light line, as shown in Figs. 3(b) and 3(c). The largest propagation length is about $3.3 \mathrm{~cm}$ for a $200 \mathrm{~nm}$-thick system. This implies that the length of the layers has to be comparable or longer than this length to enhance their thermal performance through the SPP thermal conductivity.

Figures 6(a) and 6(b) show the SPP thermal conductivity $\kappa$ as a function of the total thickness of the $\mathrm{SiO}_{2} / \mathrm{BaF}_{2}$ system surrounded by air, for three different temperatures and thicknesses, respectively. Note that when both layers of $\mathrm{SiO}_{2}$ and $\mathrm{BaF}_{2}$ are suspended in air $\left(\varepsilon_{0}=\varepsilon_{3}=1\right)$ and are of the same thickness $\left(d_{2}=0.5 d\right), \kappa$ increases with the temperature, especially when the layer thickness is scaled down. The SPP thermal conductivity reaches the phonon level for a layers
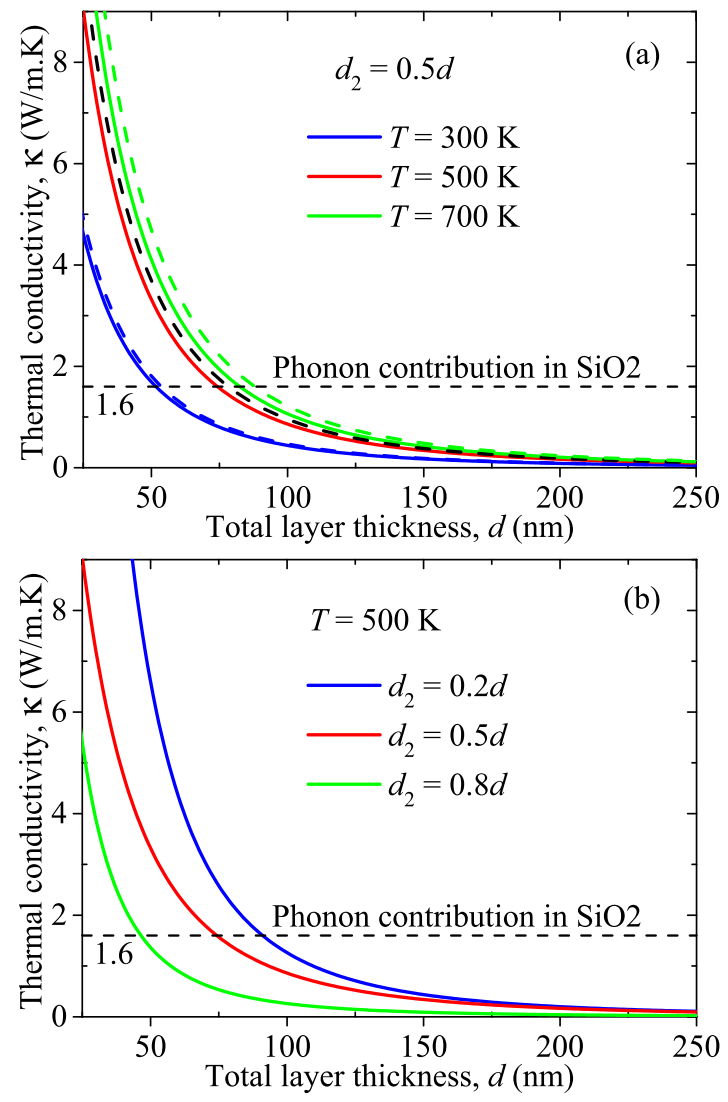

FIG. 6. Thermal conductivity as a function of total thickness of the $\mathrm{SiO}_{2} / \mathrm{BaF}_{2}$ system, for three different (a) temperatures and (b) thicknesses of the $\mathrm{SiO}_{2}$ layer. The continuous and dashed lines correspond to the cases of $\varepsilon_{0}=\varepsilon_{3}=1$ and $\varepsilon_{0}=1.24 \varepsilon_{3}=1.24$, respectively; and the phonon thermal conductivity of $\mathrm{SiO}_{2}$ applies for $T=500 \mathrm{~K}$. thickness of about $50 \mathrm{~nm}(75 \mathrm{~nm})$ at the temperature $T=300 \mathrm{~K} \quad(T=500 \mathrm{~K})$. For $d=50 \mathrm{~nm}, \kappa$ passes from $1.6 \mathrm{~W} / \mathrm{m} \cdot \mathrm{K}$ at $T=300 \mathrm{~K}$ to $3.4 \mathrm{~W} / \mathrm{m} \cdot \mathrm{K}$ at $T=500 \mathrm{~K}$ and to $4.1 \mathrm{~W} / \mathrm{m} \cdot \mathrm{K}$ at $T=700 \mathrm{~K}$, which represents enhancements of $100 \%$ and $256 \%$ with respect to the SPP thermal conductivity at room temperature. The dashed lines in Fig. 6(a) also shows that these values for the SPP thermal conductivity increases slightly when the surrounding air on one side of the layers is replaced by a substrate of $\operatorname{KBr}\left(\varepsilon_{0}=1.24 \varepsilon_{3}=1.24\right)$. This is expected given that a small difference on the permittivities $\left(\varepsilon_{0}-\varepsilon_{3}=0.24\right)$ of the surrounding media does not significantly modify the SPP dispersion relation, as shown in Eqs. (2) and (6). By properly choosing the total thickness of the layers, substrates with higher permittivities can still increase the SPP thermal conductivity, although the increase is shifted to smaller layer thicknesses. ${ }^{4,6}$ This shows that with and without the effect of the substrate, the SPPs have the potential to diminish the overheating of layered systems under high temperatures of operation, by increasing their thermal conductivity. This increase can significantly be optimized by decreasing the thickness of the absorbing layer, as shown in Fig. 6(b).

The SPP contribution to the thermal conductivity of a single- or multiple-layer system can be measured via the generation of SPPs by thermal excitation at one side of the system and detecting their diffraction at the other side, which is in yellow color in Fig. 1(a). This diffracted signal contains information about the SPP and phonon contributions to the heat flux along the layered system and it can be recorded through an IR microscope, over a wide range of frequencies and temperatures. Given that the phonon dispersion relations and the phonon thermal conductivity of a wide variety of polar materials, as $\mathrm{SiO}_{2}$ and $\mathrm{SiC}$, are well studied and reported in the literature, ${ }^{25,26}$ these experimental data are expected to allow the measurement of the SPP thermal conductivity.

\section{CONCLUSIONS}

Based on the dispersion relations predicted by the Maxwell equations, the contribution of surface phononpolaritons to the in-plane thermal conductivity of a layered system has been investigated. We have obtained an analytical expression for the thermal conductivity, which depends on the total thickness of the layers and the effective permittivity of the component layers. A thermal conductivity of $3.4 \mathrm{~W} / \mathrm{m} \cdot \mathrm{K}$ has been obtained for a two-layer system of $\mathrm{SiO}_{2}$ and $\mathrm{BaF}_{2}$, with a total thickness of $50 \mathrm{~nm}$ and at $500 \mathrm{~K}$. This thermal conductivity represents an enhancement of $100 \%$ with respect to its value for a single layer of $\mathrm{SiO}_{2}$ or for the phonon counterpart. It has been shown that higher values can be obtained by reducing the thickness of the absorbing layer of $\mathrm{SiO}_{2}$. These results show that an ensemble of layers is more efficient than one layer to enhance the heat transport by surface phonon-polaritons.

${ }^{1}$ H. J. Bakker, S. Hunsche, and H. Kurz, Rev. Mod. Phys. 70, 523 (1998). ${ }^{2}$ N. Dahan, A. Niv, G. Biener, V. Kleiner, and E. Hasman, Opt. Lett. 30, 3195 (2005).

${ }^{3}$ B. Neuner, D. Korobkin, C. Fietz, D. Carole, G. Ferro, and G. Shvets, Opt. Lett. 34, 2667 (2009).

${ }^{4}$ Dye-Zone A. Chen, A. Narayanaswamy, and G. Chen, Phys. Rev. B 72, 155435 (2005). 
${ }^{5}$ Dye-Zone A. Chen and G. Chen, Appl. Phys. Lett. 91, 121906 (2007).

${ }^{6}$ J. Ordonez-Miranda, L. Tranchant, T. Tokunaga, B. Kim, B. Palpant, Y. Chalopin, T. Antoni, and S. Volz, J. Appl. Phys. 113, 084311 (2013).

${ }^{7}$ M. Francoeur, M. P. Menguc, and R. Vaillon, Appl. Phys. Lett. 93, 043109 (2008).

${ }^{8}$ P. O. Chapuis, M. Laroche, S. Volz, and J. J. Greffet, Appl. Phys. Lett. 92, 201906 (2008).

${ }^{9}$ K. Torii, M. Ono, T. Sota, T. Azuhata, S. F. Chichibu, and S. Nakamura, Phys. Rev. B 62, 10861 (2000).

${ }^{10}$ P. O. Chapuis, S. Volz, C. Henkel, K. Joulain, and J. J. Greffet, Phys. Rev. B 77, 035431 (2008).

${ }^{11}$ N. Ocelic and R. Hillenbrand, Nature Mater. 3, 606 (2004).

${ }^{12}$ H. C. Kim and X. Cheng, Opt. Lett. 35, 3748 (2010).

${ }^{13}$ J. J. Greffet, R. Carminati, K. Joulain, J. Mulet, S. Mainguy, and Y. Chen, Nature 416, 61 (2002).

${ }^{14}$ T. Taubner, F. Keilmann, and R. Hillenbrand, Nano Lett. 4, 1669 (2004).

${ }^{15}$ E. Rousseau, A. Siria, G. Jourdan, S. Volz, F. Comin, J. Chevrier, and J. Greffet, Nat. Photonics 3, 514 (2009).
${ }^{16}$ Dye-Zone A. Chen and G. Chen, Front. Heat Mass Transfer 1, 023005 (2010).

${ }^{17}$ J. Mendialdua, A. Rodriguez, M. More, A. Akjouj, and L. Dobrzynski, Phys. Rev. B 50, 14605 (1994).

${ }^{18}$ A. Dereux, J. Vigneron, P. Lambin, and A. Lucas, Phys. Rev. B 38, 5438 (1988).

${ }^{19}$ L. Dobrzynski, Phys. Rev. B 37, 8027 (1988).

${ }^{20}$ E. Albuquerque and M. Cottam, Polaritons in Periodic and Quasiperiodic Structures (Elsevier, Amsterdam, 2004).

${ }^{21}$ P. Lambin, J. Vigneron, A. Lucas, P. Thiry, M. Liehr, J. Pireaux, R. Caudano, and T. Kuech, Phys. Rev. Lett. 56, 1842 (1986).

${ }^{22}$ E. D. Palik, Handbook of Optical Constants of Solids (Academic Press, Orlando, Florida, 1985).

${ }^{23} \mathrm{P}$. Yu and M. Cardona, Fundamentals of Semiconductors (Elsevier, London, 2010).

${ }^{24}$ V. M. Agranovich and V. Kravtsov, Solid State Commun. 55, 85 (1985).

${ }^{25}$ J. Vetelino and S. Mitra, Phys. Rev. 178, 1349 (1969).

${ }^{26}$ O. Madelung, U. Rssler, and M. Schulz, Landolt-Brnstein - Group III Condensed Matter (Elsevier, 2001), Vol. 41A1a. 